\title{
Interet D'une Supplementation En Spiruline Chez Les Enfants Drepanocytaires Homozygotes A L'hopital National De Niamey. (Essai Clinique Randomise En Double Aveugle A Propos De 53 Cas)
}

\author{
Malam-Abdou B.
}

Service d'Onco-Hématologie Hôpital National de Niamey, Niger

Brah $S$.

Andia A.

Mahamane Sani MA.

Service de Médecine Interne Hôpital National de Niamey, Niger

Chefou $M$.

Ibrahim $\boldsymbol{F}$.

Service d'Onco-Hématologie Hôpital National de Niamey, Niger

Diongolé $\boldsymbol{H}$.

Service de Néphrologie Hôpital National de Zinder, Niger

Harissou A.

Service de Chirurgie Hôpital National de Zinder, Niger

Daouda I.

Service de Pédiatrie Hôpital National de Zinder, Niger

Alhousseini Maïga D.

Service de Biochimie Hôpital National de Niamey, Niger

Mahamadou $S$.

Service National de recherche sur la Drépanocytose à Niamey, Niger

doi: 10.19044/esj.2017.v13n21p208 URL:http://dx.doi.org/10.19044/esj.2017.v13n21p208

Abstract

Spirulina is a micro algae used for thousands of years due to its healing properties. We supplemented children suffering from sickle cell anemia in order to appreciate its impact on the anthropometrical and clinical parameters. It consisted of a randomized clinical double blind test which was carried during a period of 9 months. The study concerned 53 homozygotes children suffering from sickle cell anemia aged 6 months to 15 years. Also, they were visiting the National Hospital of Niamey for follow up. One group (28 children) received $5 \mathrm{~g}$ of spirulina daily, and the other group (25 
children) received placebo. The aspects studied were: the number of hospitalizations and transfusions, the vaso-occlusive crisis, the body mass index, and the splenomegalia. We found that $84.90 \%$ of patients were hospitalized at least once and $45.3 \%$ of them have already been transfused. The average number of crisis has greatly decreased in the spirulina group from 2.75 before the study to 1.18 at the end of the study. This, thus, was observed when compared to 2.44 to 1.6 in the placebo group. We also noticed a decrease of the number of hospitalizations and the number of transfusions which is less important in the group supplemented with spirulina. In conclusion, this study showed that supplementation in spirulina had a significant impact on children suffering from sickle cell anemia. This is even despite the fact that the difference between the two groups is not significant in some cases.

Keywords: Spirulina, Sickle cell anemia, clinical trial, Niamey, Niger

\section{Résumé}

Spirulina platensis, plus couramment appelée la spiruline est une cyanobactérie faisant partie des plus anciennes formes de vies terrestres (Doudou Halidou, 2008). Elle est utilisée depuis des milliers d'années pour ses innombrables vertus nutritionnelles et thérapeutiques. Nous rapportons ici l'expérience de son utilisation chez les enfants drépanocytaires dans le but d'apprécier son impact sur le plan clinique et anthropométrique. Il s'agit d'un essai clinique randomisé en double aveugle qui s'était déroulé sur une période de 9 mois. L'étude incluait 53 enfants drépanocytaires homozygotes âgés de 6 mois à 15 ans suivis au niveau du service de pédiatrie de l'Hôpital National de Niamey. L'un des groupes (28 enfants) a reçu pendant 6 mois 5 $\mathrm{g}$ de spiruline en poudre par jour en prise unique et l'autre groupe (25 enfants) du placebo. Les paramètres étudiés étaient: le nombre d'hospitalisations et de transfusions, les crises vaso-occlusives (CVO), la splénomégalie (SPM) et l'indice de masse corporelle (IMC). Durant cette période, $84,90 \%$ des enfants ont nécessité au moins une hospitalisation et $45,3 \%$ au moins une transfusion. Au total Le nombre moyen de crises a beaucoup diminué dans le groupe spiruline passant de 2,75 à 1,18 à la fin de l'étude contre 2,44 à 1,6 dans le groupe placebo pendant la même durée. On a également observé une diminution du nombre d'hospitalisations et de transfusions dans le groupe supplémenté et une amélioration de leur état nutritionnel. Notre étude montre ainsi un bénéfice de la supplémentation en spiruline chez les enfants drépanocytaires et démontre la nécessité d'élaborer des études plus larges.

Mots-Cles : Spiruline, drépanocytose, essai clinique, Niger, Niamey 


\section{Introduction}

La drépanocytose est une maladie héréditaire de l'hémoglobine, de transmission autosomique récessive. C'est la maladie génétique la plus fréquente au monde. Elle est à l'origine de 3 grandes catégories de manifestations : anémie hémolytique chronique, phénomènes vaso-occlusifs et susceptibilité extrême à infection. C'est la première maladie moléculaire identifiée il y a plus de 50 ans. Les manifestations de la maladie sont liées à un changement des propriétés physico-chimiques de l'hémoglobine malade $(\mathrm{HbS})$ du fait de remplacement d'un acide aminé de charge négative (l'acide glutamique) par un autre de charge neutre (la Valine) en position 6 sur la chaine béta de la globines (Labie \& Elion, 2003).

La morbidité de la drépanocytose est très importante en Afrique subsaharienne. Au Niger, le trait drépanocytaire affecte 18 à 22\% soit une moyenne de $20 \%$ de la population. La drépanocytose est un véritable problème de santé publique et sa prise en charge repose en grande partie sur la gestion des crises vaso-occlusives.

Ces dernières années, la spiruline a suscité beaucoup d'intérêt comme supplément alimentaire ou comme traitement dans beaucoup de maladies. Elle est reconnue pour sa richesse en protéine et oligo-éléments ainsi que pour vertus nutritionnelles et thérapeutiques. Elle est utilisée dans la réhabilitation nutritionnelle, pour le renforcement du système immunitaire, et dans la protection contre le cancer et l'HTA (Falquet \& Hurni, 2006; Qureshi et al., 1996; Girardin-Andréani, 2005).

Cette étude a pour objectif d'évaluer l'intérêt de l'utilisation de la spiruline chez les drépanocytaires. Patients et Méthodes

Le cadre d'étude était le service de pédiatrie de l'Hôpital National de Niamey. Elle a concerné les patients drépanocytaires vus en consultation hebdomadaire par le même médecin. L'étude s'est déroulée en ambulatoire du 3 avril 2015 au 30 janvier 2016 soit une période de 9 mois dont 3 mois consacrés au recrutement et 6 mois pour le suivi.

Il s'agit d'un essai clinique randomisé en double aveugle chez des patients drépanocytaires homozygotes connus. Le projet d'étude a préalablement fait l'objet de présentation devant le Comité d'Etique National et a reçu l'autorisation de ce dernier. Un document de consentement éclairé a été expliqué et remis à chaque parent (Ces derniers signent le document avant l'inclusion des patients dans l'étude). Les parents ont été rassurés qu'ils peuvent se retirer de l'étude quand ils le désirent sans aucun risque pour le suivi de leur enfant. L'étude incluait les enfants drépanocytaires homozygotes âgés de 6 mois à 15 ans. Un examen clinique initial minutieux est effectué en vu de ne pas inclure les patients présentant un foyer infectieux quelconque. Un numéro est alors attribué de manière aléatoire au patient par l'examinateur. 
I- $\quad$ La spiruline provenait du site de culture artisanale de Koudougou au Burkina Faso. La forme utilisée est en poudre conditionnée dans des sacs de $25 \mathrm{~kg}$. Pendant la phase préparatoire, nous avons reconditionné la spiruline en poudre dans des petits sachets à la dose de $5 \mathrm{~g}$ par sachet à l'aide d'une balance de précision. Un second reconditionnement dans un grand sachet est réalisé avec 60 petits sachets de $5 \mathrm{~g}$.

La même procédure a été appliquée au placebo utilisé. La préparation du placebo a été faite à partir de la farine de mil colorée à l'aide d'un colorant alimentaire afin d'avoir un aspect identique à celui de la spiruline. Les grands sachets de Spiruline et du Placebo sont ensuite étiquetés, numérotés et consignés sur une liste par un collaborateur externe. Ainsi, ni les parents des patients, ni l'examinateur ne pouvaient connaître la nature des produits. L'identification des produits ne sera faite qu'au cours du traitement statistique.

II- La consultation consiste en l'interrogatoire du parent (ou tuteur), les mesures anthropométriques et l'examen physique. Les données sont notées dans une fiche d'enquête individuelle (pré testée). A la fin de la consultation, chaque enfant a bénéficié d'une quantité de produit nécessaire pour deux mois de supplémentation. La posologie et le mode d'administration ont été expliqués aux parents en raison d'une dose journalière de $5 \mathrm{~g}$ (1 petit sachet) en prise unique, mélangée avec de "la bouillie de céréales ou du lait fermenté ». La même quantité est renouvelée à chaque consultation (tous les deux mois) jusqu'à la fin du suivi. Les patients ont été suivis en ambulatoire

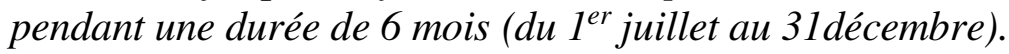

Les paramètres étudiés étaient l'âge, le sexe, la fréquence des hospitalisations, la fréquence des transfusions, la fréquence des crises vasoocclusives, la taille de la rate en millimètres mesurée à l'Echographie et l'indice de masse corporelle. Ces différents paramètres ont été déterminés sur les 6 derniers mois avant le début de l'essai et leurs moyennes ont été calculées.

Le suivi anthropométrique en vue d'apprécier l'impact de la spiruline sur l'état nutritionnel a consisté au calcul de l'indice de masse corporelle selon la formule suivante : IMC $=$ Poids $(\mathrm{kg}) /$ taille $\left(\mathrm{m}^{2}\right)$. Le poids était relevé à l'aide d'une balance type Salter d'une précision de $100 \mathrm{~g}$ et la taille mesurée à l'aide d'une toise d'une précision de $1 \mathrm{~mm}$. L'indice a été déterminé à l'inclusion, puis tous les deux mois.

Le suivi a duré 6 mois : du $1^{\text {er }}$ Juillet (début de l'administration de la Spiruline et du Placebo) au 31 décembre 2016 (fin de l'étude). Il a consisté à relever tous les 2 mois les paramètres suivants: le nombre de CVO, le nombre d'hospitalisations, le nombre de transfusions sanguines, la taille de la rate et l'indice de masse corporelle. 


\section{Résultats}

\section{Caractéristiques de L'échantillon}

Un total de 61 drépanocytaires était initialement inclus dans l'étude dont 1 cas de décès et 2 perdus de vue. Nous avions pris en compte 58 enfants restés jusqu'à la fin de l'étude dont 28 dans le groupe Spiruline et 25 dans le groupe Placebo. On note une prédominance masculine dans le groupe Spiruline et une prédominance féminine dans le groupe Placebo avec respectivement des sex ratio de 1,33 et 0,92. Les enfants de moins de 5 ans représentaient $60 \%$ ( 28 cas) et ceux de plus de 5 ans $40 \%$ ( 23 cas) avec une moyenne d'âge de 6,4 ans.

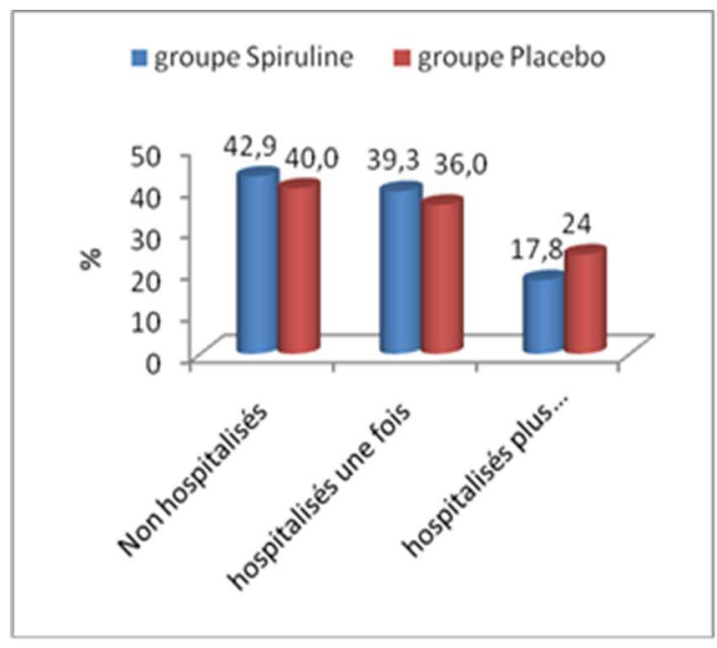

1a. Début de l'étude

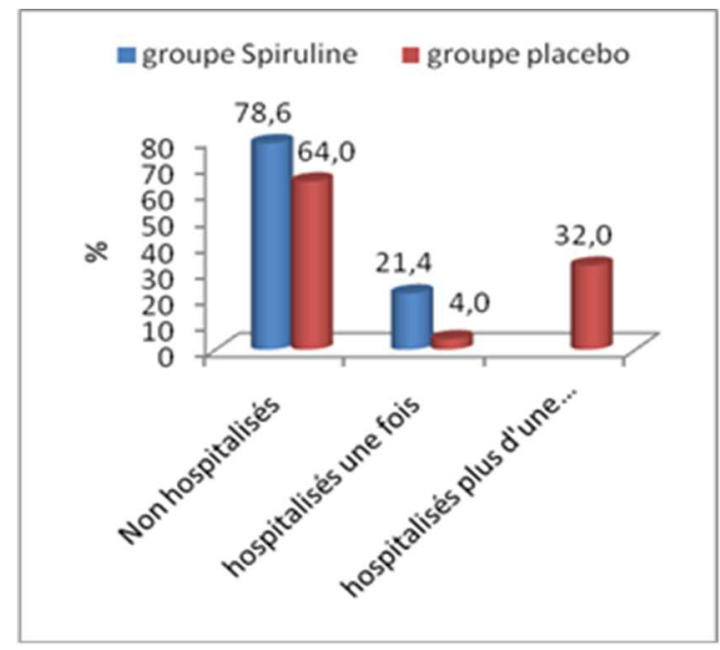

1b. Fin de l'étude

\section{Effets de la Spiruline Sur La Fréquence des Hospitalisations}

La proportion d'enfants non hospitalisés pendant la période de l'étude a presque doublé dans le groupe Spiruline (de 42,9\% à 78,6\%). Dans le groupe Placebo, elle est passée de $40 \%$ à $64 \%(\mathrm{P}=0,23)$. Le nombre d'enfants hospitalisés plus d'une fois est passé de $17,8 \%$ à $0 \%$ dans le groupe Spiruline et de 24 à $32 \%$ dans le groupe Placebo. Cette différence constatée est statistiquement significative $(\mathrm{P}=0,004)$. 


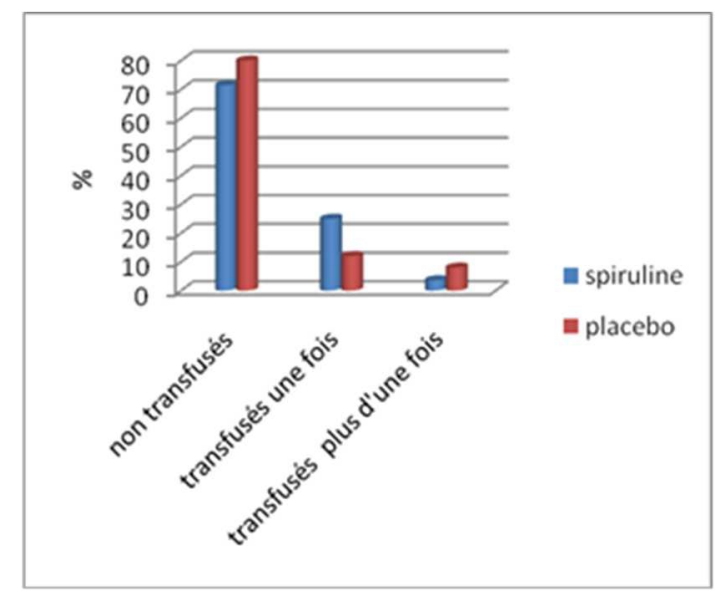

2a. Début de l'étude

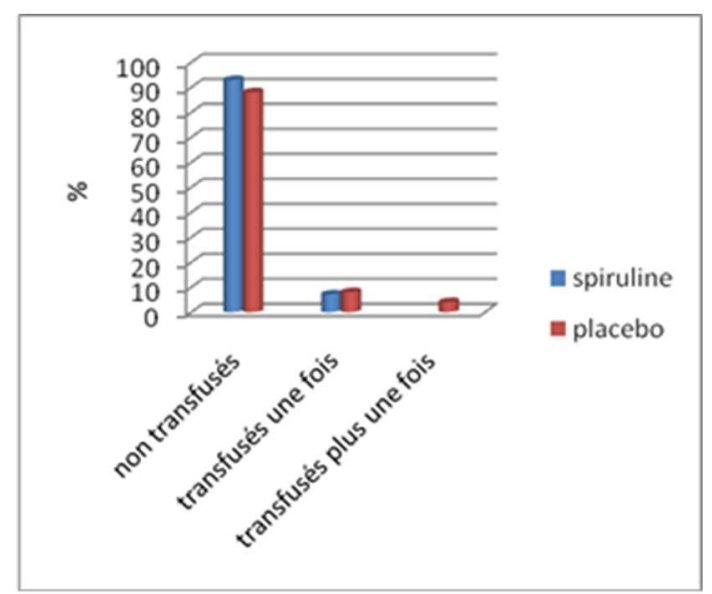

2b. Fin de l'étude

Figure 2a. Nombre de transfusions au cours des 6 mois avant l'administration de la spiruline selon les deux groupes

Figure 2b. Nombre de transfusions à la fin de l'étude selon les deux groupes

\section{Effets de la Spiruline Sur Les Crises Vaso-occlusives}

Le Tableau I montre l'évolution de la moyenne des crises vasoocclusives (CVO) dans les deux groupes. On constate une régression de la fréquence des crises beaucoup plus dans le groupe Spiruline que dans le groupe Placebo: $\mathrm{n}$ de 2,75 à 1,18 versus 2,44 à 1,60.

Tableau I. Evolution de la moyenne des crises vaso-occlusives (CVO) dans les deux groupes

\begin{tabular}{|l|c|r|}
\hline CVO & Groupe spiruline & Groupe placebo \\
\hline Moyenne au cours des 6 derniers mois & 2,75 & 2,44 \\
\hline Moyenne à la fin de l'étude & 1,18 & 1,60 \\
\hline Différence des moyennes & $-1,57$ & $-0,84$ \\
\hline
\end{tabular}

\section{Effets de la Spiruline Sur La Fréquence des Transfusions}

Le nombre d'enfants qui n'ont pas été transfusés est passé de 20 $(71,4 \%)$ avant l'etude à $26(92,9 \%)$ après l'étude dans le groupe Spiruline. Ce nombre est passé de $80 \%$ à $88 \%$ dans le groupe Placebo. Quant au nombre d'enfants transfusés plus d'une fois, il est devenu nul dans le groupe Spiruline contrairement au groupe Placebo où on note une legère regression 


\section{Effets de la Spiruline Sur L'évolution de la Splénomégalie}

La Figure 3 montre l'évolution du nombre de patients avec régression de la splénomégalie de l'inclusion à la fin de l'étude dans les deux groupes.

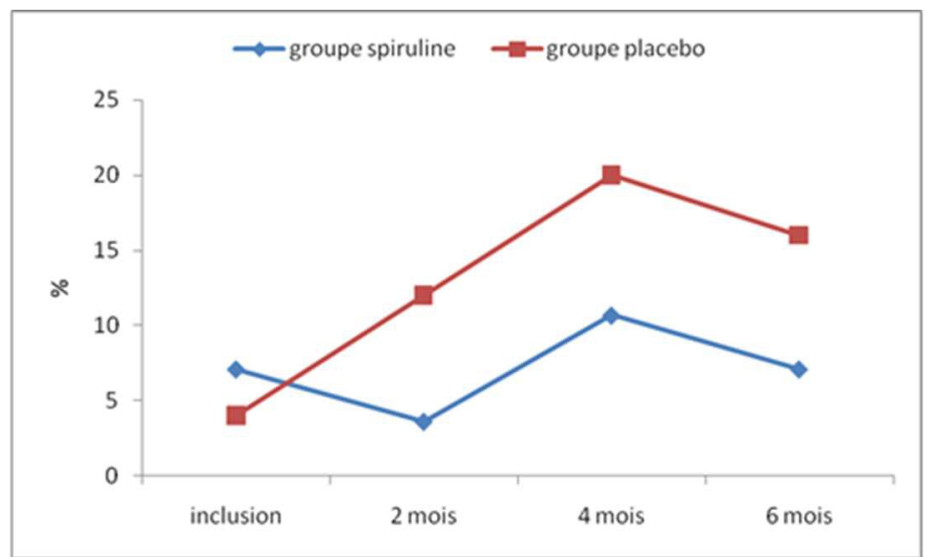

Figure 3. Evolution du nombre de patients avec régression de la splénomégalie de l'inclusion à la fin de l'étude dans les deux groupes

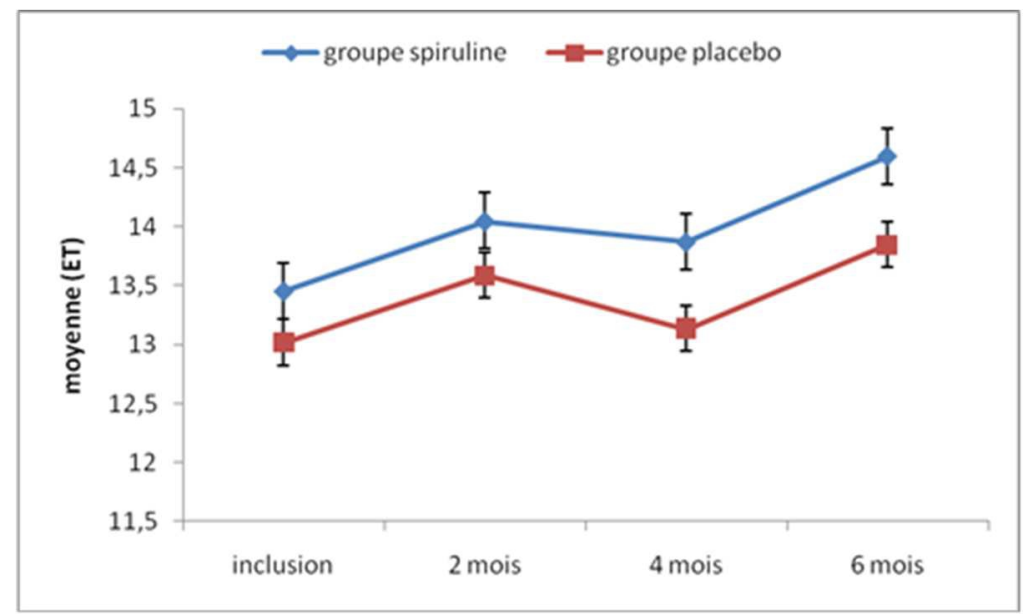

Figure 4. Evolution de l'indice de masse corporelle de l'inclusion à la fin de l'étude dans les deux groupes

Effets de la Spiruline Sur L'état Nutritionnel des Enfants Sur la Figure 4, on constate une légère élévation de l'IMC dans le groupe Spiruline par rapport au groupe Placebo. Cependant la différence n'est pas statistiquement significative.

\section{Discussion}

Malgré les quelques difficultés rencontrées au cours de cette étude d'intervention, la qualité des données collectées nous laisse penser que nos résultats obtenus sont conformes aux objectifs fixés. 
La comparaison entre les deux groupes montre des différences évidentes mais statistiquement non significatives dans la majorité des cas. Cet état de fait pourrait bien s'expliquer par la taille des échantillons dans les groupes.

Au cours du suivi, le nombre moyen de crise a diminué dans le groupe Spiruline par rapport au groupe placebo 2,75 à 1,18 crises versus 2,44 à 1,60. Ce qui a eu pour conséquence une diminution du nombre d'hospitalisations. Le nombre d'enfants hospitalisés plus d'une fois a diminué significativement dans le groupe Spiruline par rapport au groupe placebo.

Spirulina platensis est riche en vitamines du groupe $\mathrm{B}$, acides gras essentiels du groupe des oméga 6, bêtacarotène des éléments qui ont une propriété anti infectieuse par un renforcement du système immunitaire (Girardin-Andréani, 2005).

Les drépanocytaires ont une sensibilité accrue aux infections et ces infections sont le principal facteur déclenchant des CVO à l'origine d'hospitalisations et de thérapie transfusionnelle. L'amélioration du nombre moyen de crises et la réduction de la fréquence d'hospitalisations observées au cours de notre étude dénoteraient des qualités de cette algue à stimuler le système immunitaire (Qureshi \& Ali, 1996; Qureshi et al., 1996; GirardinAndréani, 2005). On note aussi une diminution importante du nombre de transfusion dans le groupe spiruline pouvant être expliquée par la richesse en Vitamine B12 de la spiruline.

On objective une discrète augmentation de la moyenne des IMC dans le groupe spiruline. Les valeurs nutritives de la spiruline pourraient bien en être l'explication. En effet, la forte teneur en substrats énergétiques (protéines, lipides, glucides) et en micronutriments (vitamines) a été prouvée par Falquet (Falquet \& Hurni, 2006; Girardin-Andréani, 2005). Des études menées par BUCAILLE P au Zaïre, Habou O et Doudou HM au Niger et Sall M.G et al au Sénégal ont montré une bonne évolution pondérale dans le cadre de la récupération nutritionnelle des enfants atteints de malnutrition sévère (Bucaille, 1992; Habou, 2003; Doudou Halidou, 2008; Sall et al., 1999).

Concernant la splénomégalie une légère régression a été constatée dans les deux groupes. La moyenne de la taille de la rate, est passée de 7,20 cm à 6,97 $\mathrm{cm}$ pour le groupe spiruline et de $6,77 \mathrm{~cm}$ à $6,54 \mathrm{~cm}$ dans le groupe placebo.

\section{Conclusion}

Au terme de cette étude, il ressort que la supplémentation en spiruline à un effet bénéfique chez les enfants drépanocytaire. L'absence de différences significatives au niveau des résultats peut être expliquée par la 
taille de l'échantillon, d'où la nécessité d'études plus larges permettant d'appréhender l'intérêt de cette microalgue chez les patients drépanocytaires.

\section{References:}

1. Ancelle T. (2006). Statistique Epidémiologique, Collection science fondamantale, $2^{\mathrm{e}}$ edition Maloine, $300 \mathrm{P}$.

2. Association de Lutte Contre la Drépanocytose à Madagascar (2008). Prise en charge de la drépanocytose dans l'Océan Indien. Colloque de l'Océan Indien Sur la drépanocytose, 57p.

3. Bucaille P. (1992). Intérêt et efficacité de l'algue spiruline dans l'alimentation des enfants présentant une malnutrition protéinoénergétique en milieu tropical (à propos de 28 observations). Thèse de doctorat en médecine. Univ. Paul Sabatier Toulouse III, Toulouse, France.

4. Doudou Halidou M. (2008). Impact d'une supplémentation en spiruline chez des enfants malnutris sévères dans le cadre de la réhabilitation nutritionnelle. Thèse de doctorat en sciences médicales, ULB, Belgique.

5. Falquet J. \& Hurni JP (2006). Spiruline: Aspect nutritionnel de la spiruline. Antenna technologies. Genève; $41 \mathrm{p}$.

6. Girardin-Andréani C. (2005). Spiruline : système sanguin, système immunitaire et cancer. Phytothérapie; 4: 158-161.

7. Habou O. (2003). Evaluation de l'efficacité de la supplémentation en spiruline du régime habituel des enfants atteints de malnutrition protéino-énergétique sévère (A propos de 56 cas), Thèse de doctorat en médecine, univ Abdou Moumouni de Niamey, Niger.

8. Kumudha A, Kumar SS, Thakur MS, Ravishankar GA, \& Sarada R (2010). Purification, Identification, and Characterization of methylcobalamin from Spirulina platensis. J. Agric. Food Chem., 58 (18), pp 9925-9930.

9. Labie D. \& Elion J. (2003). Génétique et physiopathologie de la drépanocytose in "La drépanocytose" Robert Girot, Pierre Begué, Frédéric Galactéros, Edition John Libbey Eurotext, 1-26, p322.

10. Qureshi M. A. \& Ali R.A (1996). Phagocytic potential of feline macrophages after exposure to a water soluble extract of spirulina in vitro. Immunopharmacologie.

11. Qureshi M.A Garlich J.D, Kidd M.T (1996). Dietary spirulina platensis enhances humoral and cell mediate immune functions in chickens.Immunotoxicologie.

12. Sall M.G et al. (1999). Résultats d'un essai de réhabilitation nutritionnelle avec la spiruline à Dakar (à propos de 59 cas). Médecine d'Afrique noire, 46 (3) :143 -146. 